\title{
Introducción de los editores ${ }^{1}$
}

\section{Editors introduction}

\author{
Borja Barragué \\ Universidad Nacional de Educación a Distancia - UNED (España) \\ ORCID: https://orcid.org/0000-0002-0788-1707 \\ bbarrague@der.uned.es
}

\section{NOTA BIOGRÁFICA}

Borja Barragué es profesor de filosofía jurídica y política en la Universidad Nacional de Educación a Distancia (UNED). Sus principales ámbitos de investigación son la filosofía política, las políticas de garantía de ingresos y las teorías de la justicia (contemporáneas). Su último libro es "Larga vida a la socialdemocracia".

José A. Noguera

Universitat Autònoma de Barcelona (España) ORCID: https://orcid.org/0000-0003-0263-8908

jose.noguera@uab.cat

\section{NOTA BIOGRÁFICA}

José A. Noguera es Profesor Titular de Sociología en la Universitat Autònoma de Barcelona y director del Grupo de Sociología Analítica y Diseño Institucional. Investiga sobre políticas de garantía de rentas, teoría sociológica y experimentación conductual. Ha publicado en Social Policy \& Society, Journal of Consumer Culture, Plos One, Journal of Law \& Society o Advances in Complex Systems.

Este número especial de la revista se dedica al análisis y crítica de los nudges, entendidos como intervenciones de política pública orientadas a alterar el comportamiento de los individuos sin eliminar ninguna de las opciones a su alcance ni alterar sus incentivos económicos (Thaler y Sunstein, 2009: 6). Un nudge, para poder ser calificado como tal, no puede consistir en un mandato (que lleve aparejada una sanción, típicamente jurídica) y tiene que ser además fácil de esquivar. Por poner dos ejemplos: colocar la comida más sana a la vista en un supermercado es un nudge, mientras que prohibir la venta de alcohol no lo sería.

Los nudges han tenido un éxito notable, tanto en el ámbito académico (el libro de Thaler y Sunstein en que se presenta la idea se ha convertido en un auténtico best-seller, a pesar de contener un lenguaje y una forma de presentar los argumentos más bien académica, y cuenta con más de 18.000 citas en Google Scholar en el momento en que se escribe esto), como en el institucional. En este plano, ya en 2009 el gobierno de Barack Obama reclutó a Cass Sunstein para dirigir la Oficina de Información y Asuntos Regulatorios (OIRA, por sus siglas en inglés) $)^{2}$. En 2010, el Reino Unido estableció la primera nudge unit propiamente dicha,

1 El presente número monográfico se ha visto beneficiado de los proyectos RED2018-102761-T ("El nudging aplicado a la mejora de la regulación: derecho y políticas públicas conductuales"), RED2018-102385-T ("Red de investigación en ciencias sociales experimentales"), CSO2015-64740-R ("Políticas públicas conductuales: aplicaciones a la lucha contra la pobreza, la fiscalidad y la salud alimentaria"), PID2019-104801RB-I00 ("Innovaciones en programas de garantía de rentas: políticas, pilotos y propuestas", todos dentro del Plan Nacional de I+D+i del Ministerio de Ciencia e Innovación.

2 A diferencia de otras, la OIRA no fue creada ex novo como nudge unit, sino que tiene sus orígenes en la (poéticamente bautizada como) Paperwork Reduction Act de 1980. Sunstein resumió su experiencia como regulatory czar al frente de la OIRA entre 2009 y 
la Behavioural Insights Unit (BIT), dependiente del gabinete del Primer Ministro. Muy poco después otros países le siguieron, incluyendo Australia, Canadá, Alemania, Países Bajos, Dinamarca, Noruega, Irlanda o Grecia. Además, muchos organismos multilaterales, como el Banco Mundial, la OCDE y Naciones Unidas han implementado nudge units de apoyo a sus programas ${ }^{3}$.

Ahora bien, no todas las intervenciones destinadas a influir y, en su caso, alterar el comportamiento son nudges. Sabemos, desde hace mucho tiempo, que es posible influir el comportamiento de las personas para modificarlo en un determinado sentido. De hecho, es algo que se viene haciendo tanto desde el sector público como desde el privado (sólo hay que pensar en la publicidad, por ejemplo ${ }^{4}$ ).

La tipología de Bemelmans-Videc y otros (1998) distingue tres instrumentos de política: palos, zanahorias y sermones. Los palos buscan orientar el comportamiento mediante regulaciones y sanciones, las zanahorias mediante incentivos económicos y los sermones a través de la información o persuasión. Lo que distingue, por tanto, a los nudges de otros instrumentos de política más tradicionales es que no limitan el número de cursos de acción disponibles mediante la prohibición, ni alientan un determinado comportamiento incentivándolo económicamente, ni tampoco transmiten determinada información empleando para ello una retórica más o menos persuasiva, sino que se basan expresamente en los hallazgos provenientes de la psicología cognitiva y la economía del comportamiento acerca de la racionalidad limitada de los seres humanos y los sesgos cognitivos que afectan sistemáticamente a sus creencias, preferencias y juicios.

A pesar de lo que pueda sugerir la concisa definición ofrecida por Thaler y Sunstein ya mencionada, no todos los nudges son iguales. A partir de la publicación del libro de Daniel Kahneman titulado Thinking, Fast and Slow, es habitual distinguir dos tipos de procesos cognitivos: los del sistema 1, que son rápidos y automáticos, y los del sistema 2, más lentos y reflexivos (Kahneman, 2011) ${ }^{5}$. Cuando las personas cerramos la puerta con llave al salir de casa está actuando el Sistema 1. Cuando dividimos el coste total de la suma de 8 comensales para obtener la parte alícuota, es el Sistema 2 el que toma el mando.

Tomando como base esta distinción, ha hecho fortuna una tipología que distingue dos tipos de nudges ${ }^{6}$. Los nudges no educativos o automáticos del Sistema 1 y los educativos o reflexivos del Sistema 2. Los primeros se caracterizarían por funcionar de manera automática, inconsciente y sin requerir de un esfuerzo activo por parte del individuo para alterar su comportamiento de manera efectiva. Los segundos operarían a través de la conciencia y la deliberación, siendo, por tanto, más transparentes y demandando cierto esfuerzo por parte de los agentes para funcionar.

En el artículo que abre el número, Elena Costas y Pablo Tucat ofrecen una panorámica sobre la idea de nudge y sus fundamentos en las ciencias del comportamiento, así como sobre el modo de diseñar correctamente un nudge a la hora de aplicar esta opción en el campo de las políticas públicas. Para ello, examinan dos principios básicos de los nudges como son la no reducción de las opciones de elección (el llamado «paternalismo libertario»), y la coherencia entre el nudge y la respuesta buscada. El artículo aborda cuestiones prácticas tan relevantes como la metodología de las intervenciones basadas en nudges (centrándose en la llamada metodología EAST: Easy, Attractive, Social y Timely), y finalmente, la evaluación de los mismos y de su efectividad.

En el siguiente artículo, Júlia de Quintana aborda las críticas normativas o éticas contra lo que denomina nudge-ends (es decir, los objetivos y legitimidad de los nudges) y nudge-means (el tipo de influencia de los nudges). El artículo explora las limitaciones de las críticas normativas más importantes desde ambas perspectivas y sostiene que no hay nada inherentemente incorrecto en los nudges. Las principales conclusiones del artículo son que las críticas normativas a los nudges deberían ir más allá de las implicaciones que éstos tienen dentro de la teoría del paternalismo libertario de Thaler y Sunstein, explorar cómo los distintos tipos de nudges (i. e. los automáticos y los reflexivos) afectan el comportamiento y discutir sus posibles inconvenientes más allá de las nociones de racionalidad y reflexividad a la hora de tomar decisiones.

2012, impulsando regulaciones destinadas a mejorar la alimentación en las escuelas públicas, simplificar las solicitudes de préstamos estudiantiles o implantar etiquetados tipo Nutriscore para la comida en su libro titulado Simpler (Sunstein, 2013).

3 De acuerdo con la OCDE, existen 202 instituciones incorporando los conocimientos de las ciencias del comportamiento a las políticas públicas en todo el mundo. Vid. https://www.oecd.org/gov/regulatory-policy/behavioural-insights.htm

4 Thaler y Sunstein observan, en este sentido, que hay muchas personas que hacen nudging de forma diaria seguramente de manera inconsciente y concluyen con ironía que "[i]f you are a salesperson, you are a choice architect (but you already knew that)» (Thaler y Sunstein, 2009: 3).

Como reconoce el propio Kahneman, la terminología (Sistema 1 y Sistema 2) no es suya, sino que la toma de Stanovich y West (2000).

6 Cfr. Sunstein (2016). 
GAPP. Nueva Época - N. ${ }^{2}$ 25, marzo 2021 - ISSN: 1989-8991 - DOI: https://doi.org/10.24965/gapp.i25.10918 - [Págs. 5-7] Número monográfico - Los nudges y el diseño conductual de políticas públicas

Introducción de los editores

En el tercer artículo, Antonio Cabrales y Pedro Rey Biel analizan los nudges dentro del marco de las políticas públicas basadas en la evidencia proveniente de las ciencias del comportamiento. Cabrales y Rey afirman que, para avanzar en la modernización de la administración pública hacia un mayor uso de las políticas públicas basadas en la evidencia científica, es necesario abrazar de forma más ambiciosa el enfoque de la Economía del comportamiento y no ceñirse al uso de nudges, sino incluir también intervenciones en forma de incentivo (carrots) basadas en el amplio espectro de las motivaciones que guían el comportamiento humano.

En este mismo sentido, Luis Miller sostiene en el cuarto artículo que las nuevas propuestas que encontramos en la caja de herramientas de las ciencias del comportamiento, los nudges en nuestro caso, son más bien complementarias y no un sustituto de las más tradicionales basadas en incentivos y regulaciones. Miller presenta tres tipos de ejemplos de aplicación de políticas basadas en la evidencia proveniente de las ciencias del comportamiento, en situaciones que van incorporando un grado creciente de complejidad: situaciones en que nos enfrentamos a problemas de decisión individual primero, situaciones en que hemos de tener en cuenta a otras personas después y, por último, situaciones donde existe interacción estratégica con otras personas.

Por su parte, en el quinto artículo, Julio Ponce, Estrella Montoliu y José Andrés Rozas adoptan la perspectiva del Derecho conductual para examinar las implicaciones jurídicas y lingüísticas de los nudges. Desde una visión del Derecho como sistema de regulación de conductas que va más allá de las normas jurídicas formalizadas, los autores analizan cómo los factores y mecanismos estudiados por las ciencias del comportamiento pueden tener un papel importante a la hora de entender los niveles de cumplimiento social de las normas jurídicas, así como en el diseño de las mismas y en otras muchas intervenciones destinadas a optimizar su comprensión lingüística por parte de la ciudadanía (como, por ejemplo, la simplificación del lenguaje administrativo a la hora de emitir notificaciones y publicaciones administrativas). Además, nos proponen una interesante traducción al castellano del término nudges como «acicates».

Finalmente, no podía faltar en este número una contribución sobre la utilidad del enfoque de los nudges en la gestión de la presente situación de pandemia mundial: el artículo de Hugo Cuello pone broche al monográfico abordando esta cuestión. Frente a restricciones de conducta que en este tipo de situaciones pueden ser difíciles de hacer cumplir por la vía tradicional de las prohibiciones y sanciones, la opción de los nudges puede ser una interesante herramienta complementaria para prevenir los contagios y «empujar» a la ciudadanía a seguir las recomendaciones sanitarias. El texto, cuya relevancia actual resulta evidente, ilustra su argumento con algunos ejemplos de nudges aplicados a las conductas de distancia social e higiene, discutiendo algunas de las condiciones de su efectividad.

En suma, los artículos seleccionados constituyen una panorámica actual de las discusiones sobre la estrategia del nudging en las políticas públicas, y de algunos de los campos en los que pueden aplicarse, sin olvidar el análisis crítico de sus posibles limitaciones y problemas. En un contexto de creciente demanda social y política de efectividad en la acción de las administraciones públicas, así como de evaluación rigurosa de sus actuaciones y políticas, no nos cabe duda de que la perspectiva conductual introducida por los nudges jugará un rol creciente también en nuestro país. A ello pretende contribuir, en la medida de sus posibilidades, el presente número monográfico.

\section{REFERENCIAS}

Bemelmans-Videc, M. L., Rist, R. C. y Vedung, E. (1998). Carrots, Sticks and Sermons: Policy Instruments and Their Evaluation. Routledge.

Kahneman, D. (2011). Thinking, Fast and Slow. Farrar, Strauss and Giroux.

Stanovich, K. E. y West, R. F. (2000). Individual differences in reasoning: Implications for the rationality debate? Behavioral and Brain Sciences, 23(5), 645-726. https://doi.org/10.1017/S0140525X00003435

Sunstein, C. (2016). People Prefer System 2 Nudges (Kind Of), Duke Law Journal, 66, 121-168. https://dx.doi. org/10.2139/ssrn.2731868

Sunstein, C. (2013). Simpler: The Future of Government. Simon \& Schuster.

Thaler, R. H. y Sunstein, C. (2009). Nudge. Improving Decisions About Health, Wealth, and Happiness. Penguin Random HOuse. 